\title{
A Test Suite Generation Method for Component Interaction Testing
}

\author{
Liangming $\mathrm{Li}$ \\ Zibo Vocational Institute, Zibo, China \\ lilm@zbvc.edu.cn
}

Keywords: Interaction testing; Test case; Component composition; Unit testing

\begin{abstract}
Composition technology is the key factor of component based software development. Assembling component models to derive interaction test cases is a common way of component interaction testing; however, one of the consequences is the difficulty to test different component models sufficiently and the other is state explosion problem. We propose a method to generate interaction testing suite based on the composition of unit test case. The proposed method is a generally-used way for interaction testing and could meet the demand of appointed test criteria.
\end{abstract}

\section{Introduction}

Component-Based Software Development (CBSD) is the process of building soft-ware system by assembling existing components. Although unit testing can validate the correctness of local module [1], interaction errors may be still existed during component composition in practice [2], so component interaction should test thoroughly and completely during integration [3].

The current researchers usually perform compositional testing by means of model combination, such as Beydeda and Gruhn [4] that use the component state machine (CSM) to describe the behavior of individual component, and then construct the component-based software flow graph (CBSFG) for generation of test cases according to the CSMS; Wu et al. [5] first utilizes the component interaction graph (CIG) to describe the interactions and the dependence among components, and then generate the test case based on the CIG.

However, this research focus on the analysis of the integrate system function and locating the interactions of model combination. The consequences are the problems of state space explosion when the models contain considerable states and the high requirement for tester to understand different models.

\section{Concepts and Terminology}

Some concepts to be used are introduced in this section and the definition is simplified to facilitate the study.

Definition 1 a component is a 3-tuple $M=\left\langle I P_{M}, I R_{M}, P_{M}\right\rangle$

$I P_{M}$ is the interface set of $M$ to provide services;

$I R_{M}$ is the interface set of $M$ to require services;

$P_{M}$ is the protocol of $M$ to describe the behavior.

Definition 2 The test suite of component $M\left(T_{M}\right)$ is the set of test cases that satisfy a user's testing requirements, which is provided by the component developer or generated by the component user according to the interface instruction and protocol. We use $t=\left(\left(i_{1}, \ldots, i_{m}\right),\left(o_{1}, \ldots, o_{n}\right)\right)$ to describe a test case in which $\mathrm{i}$ is input information and $\mathrm{o}$ is output information.

Definition 3 Two component $P$ and $Q$ are composable if:

$$
I P_{P} \cap I P_{Q}=\varnothing \quad, \quad I R_{P} \cap I R_{Q}=\varnothing \quad, \quad I P_{P} \cap I R_{Q} \neq \varnothing \quad \text { or } \quad I P_{Q} \cap I R_{P} \neq \varnothing \quad . \quad \text { We let }
$$

$\operatorname{Shared}(P, Q)=\left(I P_{P} \cap I R_{Q}\right) \cup\left(I P_{Q} \cap I R_{P}\right)$ and $P \bullet Q$ is the symbol of two component composition. 
The deadlock and illegal state are assumed will not occur when component composition which mean the assembly ability has validated at the design stage. The following definition of test criteria is mainly from reference [5].

Subdomain is defined as containing all the inputs that can cause a particular code to be executed in the subdomain policy based on the program structure. There is a program $\mathrm{P}$ contain the component $\mathrm{M}$ and a test adequacy criterion $\mathrm{C}$ based on subdomain, $\delta D_{C}\left(D_{P}\right)$ is the induced multi-set of $\mathrm{P}$ according $\mathrm{C}$, $\delta D_{C}\left(D_{M}\right)$ is the induced multi-set of $\mathrm{M}$ according $\mathrm{C}, M$-traverse $\left(D_{P}\right)$ is the input set of $\mathrm{P}$ which result in $\mathrm{M}$ is called, $M$-bypass $\left(D_{P}\right)$ is the input set of $\mathrm{P}$ which is irrelevant to $\mathrm{M}$.

Definition 4 Test suite $T_{P}$ is $C$-adequate for $\mathrm{P}$ if $T_{P}$ contain each test sample of $\delta D_{C}\left(D_{P}\right) ; T_{M}$ is $C$-adequate for $\mathrm{M}$ if $T_{M}$ contain each test sample of $\delta D_{C}\left(D_{M}\right)$.

The Test Suite Generation Method for Component Interaction. The universal model from component composition is introduced, then the correctness and effectiveness of the proposed method are theoretically analyzed, after that the implementation process of the method is illustrated.

The General Model Of Component Composition. System development is replaced by the idea of assembling existing components in the process of CBSD. The general model of component composition is shown in Fig. 1 and the composition of several components could be performed by pair-wise composition

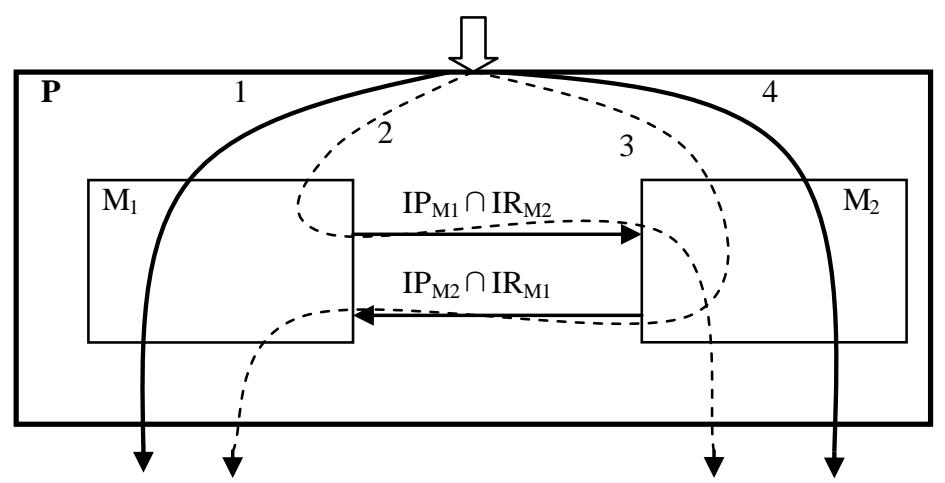

Figure 1. The model of component composition

The program $\mathrm{P}$ is constructed by composition of component $M_{1}$ and $M_{2}$ in Fig. 1 in which the line which is tagged with number 1 and 2 represent the input set of $M_{1}-\operatorname{traverse}\left(D_{P}\right)$ and the dash 2 represent the related input subdomain of $I P_{M 1} \cap I R_{M 2}$; Similarly the line 3 and 4 represent the input set of $M_{2}-\operatorname{traverse}\left(D_{P}\right)$ and dash 3 represent the related input subdomain of $I P_{M 2} \cap I R_{M 1}$.

From Fig. 1, One part of the system functions is implemented by $M_{1}$ and $M_{2}$ individually and the other functions are cooperative performed by the interaction of $M_{1}$ and $M_{2}$. If $M_{1}$ and $M_{2}$ had passed the unit testing and gotten the test suit $T_{M 1}$ and $T_{M 2}$ then line 1 and 4 in figure 1 can be tested by mapping the related part of $T_{M 1}$ and $T_{M 2}$ to input of $\mathrm{P}$. The dash line 2 represent the input of $M_{2}$ that is provided by $M_{1}$, which can be tested by composition of the related test cases in $I P_{M 1} \cap I R_{M 2}$ from $T_{M 1}$ and $T_{M 2}$, similarly the line 3 can be tested by combination of related test cases in $I P_{M 2} \cap I R_{M 1}$.

The Method of Test Suite Generation for interaction Testing. The test case set $T_{M}$ can be divided into relevant and irrelevant part for interface $I_{M}$ of component $\mathrm{M}$.

Definition $5 T_{M}-\operatorname{relevant}\left(I_{M}\right)$ : the relevant part of $T_{M}$ with component $I_{M}$

Definition $6 T_{M}$-irrelevant $\left(I_{M}\right): T_{M}-\left(T_{M}-\operatorname{relevant}\left(I_{M}\right)\right)$

Definition 7 let $t_{1}=\left(\left(i_{1}, \ldots, i_{m}\right),\left(o_{1}, \ldots, o_{n}\right)\right), t_{2}=\left(\left(i_{1}^{\prime}, \ldots, i^{\prime}{ }^{\prime}\right),\left(o_{1}^{\prime}, \ldots, o^{\prime}{ }^{\prime}\right)\right)$ the combination of $t_{1}$ and $t_{2}$ is $t_{1} \bullet t_{2}$

$t_{1} \bullet t_{2}=\left(\left(\left(i_{1}, \ldots, i_{m}\right) \cup\left(i_{1}^{\prime}, \ldots, i_{m}^{\prime}\right)-X\right),\left(\left(o_{1}, \ldots, o_{n}\right) \cup\left(o_{1}^{\prime}, \ldots, o_{n}^{\prime}\right)-X\right)\right), \forall x \in X, x \in\left(o_{1}, \ldots, o_{n}\right) \cap\left(i_{1}^{\prime}, \ldots, i_{m^{\prime}}{ }^{\prime}\right)$, in which $X \neq \varnothing$ and $t_{1} \bullet t_{2}$ represent the input of $t_{2}$ is the output of $t_{1}$. 
After the analysis, $T_{M 1}-\operatorname{relevant}\left(I P_{M 1} \cap I R_{M 2}\right)$ is the test suite of $M_{1}$ that could call the function of $M_{2}$ and $T_{M 2}$-relevant $\left(I P_{M 1} \cap I R_{M 2}\right)$ is the test suite of $M_{2}$ that could call the function of $M_{1}$. The combination of $T_{M 1}-$ relevant $\left(I P_{M 1} \cap I R_{M 2}\right)$ and $T_{M 2}$-relevant $\left(I P_{M 1} \cap I R_{M 2}\right)$ could test the activity of $I P_{M 2} \cap I R_{M 1}$. Therefor we get the interaction testing method by combination of test suite, which is IT-on-CUTC (Interaction Testing based on Combination of Unit Test Case, IT-on-CUTC), $T_{1} \# T_{2}$ is used to describe the combination of test suite $\mathrm{T}_{1}$ and $\mathrm{T}_{2}$.

The steps of IT-on-CUTC are as follows:

Premise: component $M_{1}$ and $M_{2}$, test criterion $C$ and test suite $T_{M 1}$ and $T_{M 2}$

Step1: according $I P_{M 1}, I R_{M 1}$ and $I P_{M 2}, I R_{M 2}$ to determine if $M_{1}$ and $M_{2}$ is composable and the operation will stop if $M_{1}$ and $M_{2}$ is not composable;

Step2: compute the $\operatorname{Shared}\left(M_{1}, M_{2}\right)$, namely compute the $I P_{M 1} \cap I R_{M 2}$ 与 $I P_{M 2} \cap I R_{M 1}$;

Step3: determine the $T_{M 1}-\operatorname{relevant}\left(I P_{M 1} \cap I R_{M 2}\right)$ and $T_{M 1}-\operatorname{relevant}\left(I P_{M 2} \cap I R_{M 1}\right)$ from $T_{M 1}$, determine the $T_{M 2}-$ relevant $\left(I P_{M 1} \cap I R_{M 2}\right)$ and $T_{M 2}-\operatorname{relevant}\left(I P_{M 2} \cap I R_{M 1}\right)$ from $T_{M 2}$;

Step4: compute $\quad T_{M 1}-\operatorname{relevant}\left(I P_{M 1} \cap I R_{M 2}\right) \quad \# \quad T_{M 2}-\operatorname{relevant}\left(I P_{M 1} \cap I R_{M 2}\right) \quad$ and $T_{M 2}-$ relevant $\left(I P_{M 2} \cap I R_{M 1}\right) \# T_{M 1}-\operatorname{relevant}\left(I P_{M 2} \cap I R_{M 1}\right)$ then get the union $T_{M 1 \bullet M 2}$;

Step5: analyze $T_{M 1 \bullet M 2}$ and remove the meaningless test cases; run $T_{M 1 \bullet M 2}$ and finish the interaction testing of $M_{1}$ and $M_{2}$.

Theorem 1. The test suit $T_{M 1 \bullet M 2}$ generated from IT-on-CUTC is $C$-adequate for component interaction

Proof: the composition of $M_{1}$ and $M_{2}$ do not generate new interface and no new partition is made for the input domain. Then $\left|I P_{M 1 \bullet M 2}\right|+\left|I R_{M 1 \bullet M 2}\right|<\left|I P_{M 1}\right|+\left|I R_{M 1}\right|+\left|I P_{M 2}\right|+\left|I R_{M 2}\right|$ and $\delta D_{C}\left(D_{M 1 \bullet M 2}\right) \subseteq\left(\delta D_{C}\left(D_{M 1}\right) \cup \delta D_{C}\left(D_{M 2}\right)\right)$. So the testing of $M_{1} \bullet M_{2}$ could implement in the original input domain according to the testing criteria $C$. On the other hand $T_{M 1}$ and $T_{M 2}$ is $C$-adequate for $M_{1}$ and $M_{2}$ and $T_{M 1 \bullet M 2}$ is generated by the related test cases to $\operatorname{Shared}\left(M_{1}, M_{2}\right)$ from $T_{M 1}$ and $T_{M 2}$ which could cover all the interaction path of $M_{1}$ and $M_{2}$ based on $C$. Therefor the test suit $T_{M 1 \bullet M 2}$ generated from IT-on-CUTC is $C$-adequate for interaction of $M_{1} \bullet M_{2}$.

\section{Literature References}

Weyuker E J [6] define 8 axioms to formalize the basic properties of the adequacy of test cases and the anti- decomposition and the anti-combination axiom illustrate the test adequacy between the overall procedure and the components. Frankl F G, and Weyuker E J [7] further analyze the different test criteria based on subdomain division. Rosenblum D S [5] define the testing criteria C-adequate-for-P of unit testing and $\mathrm{C}$-adequate-for-M of integration testing. LIU Ling, MIAO Huai-kou [8] define an axioms system to describe the relations among the logic coverage criteria. Some interaction testing are based on component model, Hierons R M [9] present the method using CIS(Constrained identification sequence) as state recognition sequence to test the interaction among FSM. The approach of integration testing is proposed in [10] based on UML, first, According to the model, the interaction activities of different components are merged and then the test cases are derived from the composed model. Gotzhein and Khendek [11] propose a compositional test method, which test the concurrent component composition without constructing the global state machines.

From the above survey, we find that the studies usually concern how to establish testing criteria and discuss the relation between them, which do not concentrate on describing the method of generating interaction test suite. In this paper we study the method of obtaining interaction test suite from unit test cases and prove the method could meet the test criteria. 


\section{Summary}

Interaction testing is the important part of softer testing and which is the essential way to validate component composition. The study on component interaction testing is usually based on model combination, but if the components are described by different models, the completion of interaction testing will be very difficult and inefficient when a lot works of model transformation are needed. We propose a novel interaction testing approach, called IT-on-CUTC, which derives test cases from interaction path. The method performs interaction testing with low computational complexity and more generality; therefore the development cost is reduced.

In the future, we will further optimize the IT-on-CUTC algorithm for generating interaction path more efficiently, and conduct more experiments at different scale to verify its validity and practicability.

\section{References}

[1] E.J. Weyuker, Testing Component-Based Software: A Cautionary Tale: IEEE Software, 1998, 15(5): 54-59.

[2] S. Eldh, S. Punnekkat, H. Hansson, et al, Component Testing Is Not Enough -A Study of Software Faults: Proc. 19th IFIP International Conference on Testing of Communicating Systems TESTCOM/FATES, Springer LNCS-4581, Tallinn, Estonia 2007, 74-89.

[3] S. Beydeda S, V. Gruhn. An integrated testing technique for component-based software: Proc. of the ACS/IEEE Int'1 Conf on Computer Systems and Applications. Beirut, IEEE Computer Society Press, 2001. 328-334.

[4] Y. Wu, D. Pan, M. H. Chen. Techniques for testing component-based software: Proc. of the 7th IEEE Int'1 Conf on Engineering of Complex Computer Systems(ICECCS'01). Skovde, IEEE Computer Society Press, 2001. 222-232.

[5] D. S. Rosenblum. Adequate Testing of Component-Based Software: Technical Report, UCI-ICS-97-34, University of California, Irvine. August, 1997.

[6] E. J. Weyuker. Axiomatizing software test data adequacy: IEEE Tran. on Software Engineering, 1986, 12(12):1128-1138.

[7] F. G. Frankl, E. J. Weyuker. A formal analysis of the fault- detecting ability of testing methods: IEEE Transactions on Software Engineering, 1993, 19(3): 202- 213.

[8] L. Ling, L. Huai-kou. Axiomatic Assessment of Logic Coverage Software Testing Criteria: Journal of Software, 2004,15 (9) :1301-1310.

[9] R. M. Hierons. Checking states and transitions of a set of communicating finite state machines: Microprocessors and Microsystems, 2001, 24(9): 443-452.

[10] J. Hartmann, C. Imoberdorf, M. Meisinger. UML-based integration testing: Proc. of 2000 ACM SIGSOFT international symposium on Software Testing and Analysis, Portland, Oregon, United States, 2000. 60-70.

[11]R. Gotzhein, F. Khendek. Compositional Testing of Communication Systems: Proc. of IFIP Testcom 2006, Berlin: Springer LNCS 3964, 2006. 227-244. 